\title{
Functional relationship heavy metal P-type ATPases (OsHMA 2 and OsHMA3) of rice (Oryza sativa) using RNAi
}

\author{
Namiko Satoh-Nagasawa*, Mikako Mori, Kenji Sakurai, \\ Hidekazu Takahashi, Akio Watanabe, Hiromori Akagi \\ Laboratory of Plant Genetics and Breeding, Department of Biological Production, Faculty of Bioresource Sciences, Akita \\ Prefectural University, Akita 010-0195, Japan \\ *E-mail: satohnagasawa@akita-pu.ac.jp Tel: +81-18-872-1644 Fax: +81-18-872-1678
}

Received April 15, 2013; accepted June 16, 2013 (Edited by C. Matsukura)

\begin{abstract}
Oryza sativa heavy metal P-type ATPase 3 (OsHMA3) plays a major role in the hyperaccumulation of cadmium (Cd) by the rice cultivars 'Cho-Ko-Koku (CKK)' and 'Anjana Dhan.' On the other hand, mutants that accumulate Cd in the shoots at a very low level have suggested the function of several genes such as OsHMA2 (which is neccesary to load Cd into xylem) or O. sativa natural resistance-associated macrophage protein 5 (OsNRAMP5, which regulates the uptake of $\mathrm{Cd}$ from soil into the roots). Although many studies have examined metal transporters, few have investigated the function of combination of multiple gene in plant. Herein, first, we used an RNA interference (RNAi) method to down regulate OsHMA3 and recovered plants in which the expression of OsHMA3 was strongly suppressed. Second, crossing of OsHMA3 RNAi plants with oshma2 mutants revealed a functional relationship between OsHMA3 and OsHMA2 in plant. We concluded that a complete loss of function of OsHMA2 is crucial to minimize Cd translocation from the roots to the shoots. The current findings also implied that the activity of OsHMA2 can be accelerated in plant in the roots without the C-terminal region of the protein.
\end{abstract}

Key words: Cadmium, Oryza sativa, OsHMA2, OsHMA3, RNAi.

Heavy metal pollution of soil is harmful to human health and agriculture (Clemens et al. 2012; Nriagu and Pacyna 1988; Wei and Zhou 2008). To breed new food crops that do not accumulate heavy metals, and to develop plants for phytoextraction that accumulate high levels of heavy metals, the molecular mechanisms of several key processes in heavy metal accumulation must be elucidated (Mendoza-Cozatl et al. 2011; Milner and Kochian 2008; Verbruggen et al. 2009).

Rice is a major staple crop for sustaining the world's population and a model system for monocot species, which explains why the elucidation of molecular knowledge regarding heavy metal accumulation, especially for cadmium (Cd), has recently accelerated (Clemens et al. 2012). Several studies showed that Oryza sativa natural resistance-associated macrophage protein 5 (OsNRAMP5) accounts for most of the Cd uptake from the soil by rice (Ishikawa et al. 2012; Ishimaru et al. 2012; Sasaki et al. 2012). The function of $O$. sativa heavy metal P-type ATPase 3 (OsHMA3) was revealed in the analysis of the rice cultivars 'Cho-Ko-Koku (CKK)' and 'Anjana Dhan', whose shoots accumulate high levels of $\mathrm{Cd}$.
Miyadate et al. (2011) and Ueno et al. (2010) reported that the phenotype of these two cultivars resulted from a defect in the structure of OsHMA3 that transports $\mathrm{Cd}$ into vacuoles in rice roots and limits $\mathrm{Cd}$ translocation from roots to shoots. On the other hand, insertion mutants resulting in the loss of the function of another heavy metal P-type ATPase in rice, OsHMA2 were used to demonstrate the gene function. OsHMA2 transports $\mathrm{Cd}$ and zinc $(\mathrm{Zn})$ into the xylem and is responsible for the translocation of Cd and Zn (Nocito et al. 2011; SatohNagasawa et al. 2012; Takahashi et al. 2012).

Although studies on heavy metal transporters in rice have progressed markedly, most of them focused on a single transporter. However, more information about the function of transporters may be gained when combination of genes are investigated simultaneously through genetic engineering. For example, functional redundancy or an additive effect can be revealed by such studies (Hussain et al. 2004; Ishimaru et al. 2012). Herein, we used OsHMA3 RNA interference (RNAi) plants to investigate the relationship between OsHMA3 and OsHMA2 in plant by analyzing the $\mathrm{F}_{2}$ progeny of

Abbreviations: OsHMA3, Oryza sativa heavy metal P-type ATPase 3; Cd, cadmium; CKK, Cho-Ko-Koku; RNAi, RNA interference; Zn, zinc; OsNRAMP5, Oryza sativa natural resistance-associated macrophage protein 5; NPT, neomycin phosphotransferase; HPT, hygromycin phosphotransferase; LB, left border; RB, right border; UTR, untranslated region; RT-PCR, reverse transcription polymerase chain reactio.

This article can be found at http://www.jspcmb.jp/

Published online November 14, 2013 


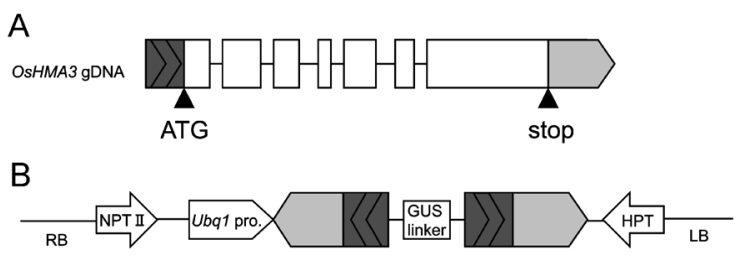

Figure 1. Structure of the RNAi construct of OsHMA3. (A) OsHMA3 and the inverted repeat region in the pANDA vector. OsHMA3 has seven exons (white boxes). Dark gray boxes are $5^{\prime}$-UTR and gray boxes are $3^{\prime}$-UTR. ATG is the translation start site. (B) The fusion between $180 \mathrm{bp}$ of the $5^{\prime}$-UTR and $250 \mathrm{bp}$ of the $3^{\prime}$-UTR of OsHMA3 in the pANDA vector. RB, right border; NPTII, neomycin phosphotransferase II; Ubq1 pro., maize ubiquitin 1 promoter; HPT, hygromycin phosphotransferase; LB, left border.

OsHMA3 RNAi plants and OsHMA2 mutants that were both in the 'Nipponbare' genetic background.

To downregulate OsHMA3 exclusively without affecting the expression of other HMA-family genes, we used the $5^{\prime}$-untranslated region (UTR) and $3^{\prime}$-UTR regions of OsHMA3 for targeting RNAi suppression (Figure 1). To create the construct, we performed three polymerase chain reactions (PCRs) with KOD Plus (Toyobo, Osaka, Japan). The first PCR was performed with primers HAF1 and P42 to amplify the $5^{\prime}$-UTR of OsHMA3, and primers P6 and P43 to amplify the $3^{\prime}$ UTR of OsHMA3. The sequences of all primers used in this study are listed on Table 1. PCR was carried out for 35 cycles of $30 \mathrm{~s}$ at $94^{\circ} \mathrm{C}, 30 \mathrm{~s}$ at $68^{\circ} \mathrm{C}$, and $30 \mathrm{~s}$ at $68^{\circ} \mathrm{C}$. For fusing the $5^{\prime}$ - and $3^{\prime}$-UTRs, a second PCR was performed with primers $\mathrm{P} 44$ and $\mathrm{P} 45$ that contain sequences overlapping with the forward attB1 and reverse attB2 Gateway primers, respectively. After the third PCR with primers P50 and P51, which also include the forward attB1 and reverse attB2 sequences, Gateway cloning into the pANDA vector (Miki et al. 2005; Miki and Shimamoto 2004) was performed as described by Mohanty et al. (2009). The pANDA-OsHMA3 RNAi construct and empty pANDA vector (control) were used for Agrobacterium tumefaciens-mediated transformation of 'Nipponbare' calli (Hiei et al. 1994). Sixteen lines containing the pANDA-OsHMA3 RNAi construct and 14 lines containing the pANDA vector were regenerated on hygromycin medium, then transferred to soil in pots in a greenhouse with temperatures ranging from 20 to $28^{\circ} \mathrm{C}$ and natural light conditions. Part of the $\mathrm{T}_{0}$ plants was used for a preliminary analysis, and the $\mathrm{T}_{1}$ seeds were collected from plants in the greenhouse.

For analyzing the genotypes of $\mathrm{T}_{0}$ and $\mathrm{T}_{1}$ plants and selecting them for further analyses, DNA was extracted from seedlings using the method of Klimyuk et al. (1993), and PCR was performed using ExTaq (TaKaRa, Shiga, Japan) with primers P45 and P61 to select plants containing the pANDA-OsHMA3 RNAi construct or pANDA vector (control). It was carried out for 35 cycles of $30 \mathrm{~s}$ at $94^{\circ} \mathrm{C}, 30 \mathrm{~s}$ at $55^{\circ} \mathrm{C}$, and $30 \mathrm{~s}$ at $72^{\circ} \mathrm{C}$.
Table 1. Primer sequences used in the study.

\begin{tabular}{ll}
\hline HAF1 & 5'-CTGCCATACACAACCATTGC-3' \\
P4 & 5'-CTGTTGAGCACGACGAGGAG-3' \\
P6 & 5'-GACACGCTTGTGGCTATTGAG-3' \\
P13 & 5'-ATTGGGAACAGAAGGACATTG-3' \\
P42 & 5'-CTTGCAACACGCCGTCGTCTCGTCCACCGCG- \\
& CCTC-3' \\
P43 & 5'-ACGACGGCGTGTTGCAAGATTGACCAGTAAT- \\
& TCAC-3' \\
P44 & 5'-GCTCGATCCACCTAGGCTCTGCCATACACAA- \\
& CCATTGC-3' \\
P45 & 5'-CGTAGCGAGACCACAGGAGACACGCTTGTGG- \\
& CTATTGAG-3' \\
P48 & 5'-GGTATCGTCAGCAACTGGGATGATATGG-3' \\
P49 & 5'-GCTCCGTCAGGATCTTCATGAGGTAATC-3' \\
P50 & 5'-GGGGACAAGTTTGTACAAAAAAGCAGGCTG- \\
\multicolumn{2}{c}{ CTCGATCCACCTAGGCT-3' } \\
P51 & 5'-GGGGACCACTTTGTACAAGAAAGCTGGGTCG- \\
\multicolumn{1}{c}{ TAGCGAGACCACAGGA-3' } \\
P61 & 5'-GTATCAGTGTGCATGGCTGG-3' \\
\hline
\end{tabular}

To analyze the expression of OsHMA3 in the $\mathrm{T}_{1}$ plants, total RNA was extracted according to the methods of Satoh-Nagasawa et al. (2012). Then, reverse transcription PCR (RT-PCR) was performed with KOD plus (Toyobo) and primers P4 and P13. Actin (LOC_OS03G50885.1) cDNA amplified with Ex Taq (TaKaRa) and primers P48 and P49 were used as the standard (Sasaki et al. 2012; Satoh-Nagasawa et al. 2012). PCR was performed for 28 and 29 cycles for OsHMA3 and 23 and 24 cycles for Actin. The PCR program was the same as that above. Quantity One (Bio-Rad, Hercules, CA, USA) was used to measure the band volumes to ensure that CDNAs were exponentially amplified. The relative OsHMA3 expression levels measured by RT-PCR were normalized to the Actin control.

Plants obtained by RNAi showed several patterns of downregulation of OsHMA3. In lines R3 and R6, the expression of OsHMA3 was highly suppressed, whereas in line R8, the suppression was only partial; in line R13, OsHMA3 expression was not suppressed (Figure 2A). For statistical analysis, JMP 5.1.2 was used (Tukey method; SAS Institute, Cary, NC, USA).

With the aim of investigating the effect of suppressing OsHMA3 expression, selected $\mathrm{T}_{1}$ plants containing the RNAi constructs and controls were grown hydroponically. Rice seeds were surface sterilized, incubated, and cultured for 27 days according to the method of Satoh-Nagasawa et al. (2012). For Cd treatment, all seedlings were grown hydroponically in a nutrient solution containing $5 \mu \mathrm{g} \mathrm{l}^{-1} \mathrm{CdCl}_{2}$ for 3 weeks. The nutrient solution was renewed every 2 days, and $\mathrm{CdCl}_{2}$ was added daily. All experiments were performed in a growth chamber $\left(14 \mathrm{~h}\right.$ of light at $26^{\circ} \mathrm{C}$ and $10 \mathrm{~h}$ of darkness at $22^{\circ} \mathrm{C}$ ). Following the hydroponic culture period, the $\mathrm{Cd}$ content was measured according to the method of Satoh-Nagasawa et al. (2012). The 

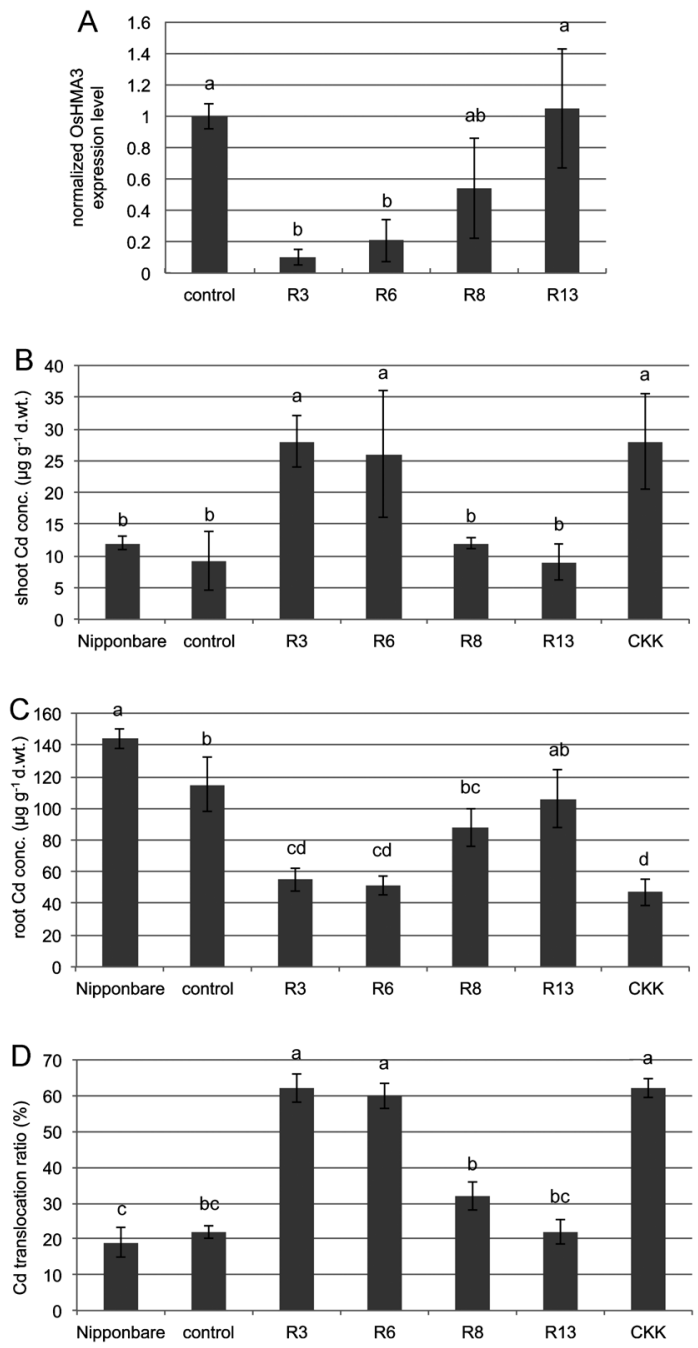

Figure 2. RNA silencing of OsHMA3 estimated with semiquantitative RT-PCR and the phenotype of the RNAi $\mathrm{T}_{1}$ lines for Cd accumulation. (A) Normalized OsHMA3 expression level in four RNAi $\mathrm{T}_{1}$ lines; (B) shoot Cd concentration; (C) root Cd concentration; (D) Cd translocation ratio. The data for untransformed Nipponbare, the empty pANDA vector (control), and CKK are included. Vertical bars represent the standard deviation of four plants. Letters above the bars indicate the level of significance at $p<0.05$ using the Tukey method.

translocation ratio of metals from the root to the shoot was estimated as the percentage of the metals in the shoot compared to that in the whole plant.

Line R3 in which OsHMA3 expression was well suppressed had a high concentration of $\mathrm{Cd}$ in the shoots and a low concentration of $\mathrm{Cd}$ in the roots, resulting in a high $\mathrm{Cd}$ translocation ratio. In contrast, $\mathrm{Cd}$ accumulation in line R13 in which OsHMA3 expression was not suppressed was exactly the opposite (Figure 2). Note that in both cases, the concentrations of $\mathrm{Cd}$ in shoots and the $\mathrm{Cd}$ translocation ratios corresponded quite well with each other, as observed in our previous study (Satoh-Nagasawa et al. 2012). The results showed that OsHMA3 is responsible for translocating $\mathrm{Cd}$ into the shoots. To determine whether OsHMA3 is involved in the translocation of other metals, we
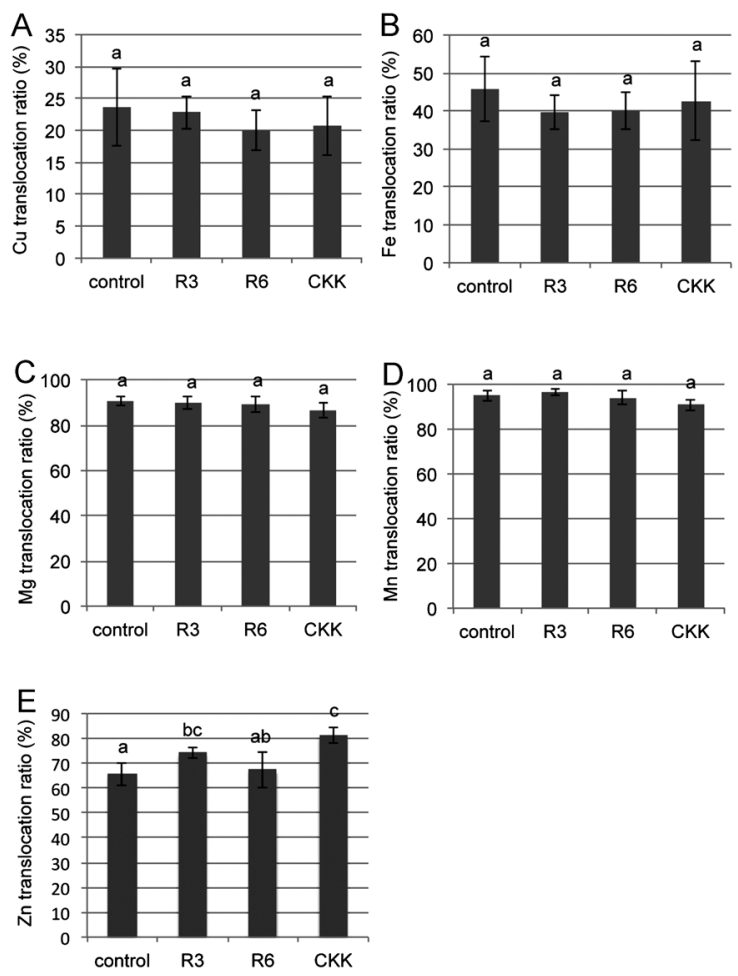

Figure 3. Translocation ratios of metals in OsHMA3 RNAi $\mathrm{T}_{1}$ lines, the control, and CKK. Translocation ratios of (A) $\mathrm{Cu}$, (B) Fe, (C) $\mathrm{Mg}$, (D) $\mathrm{Mn}$, and (E) $\mathrm{Zn}$ when $5 \mu \mathrm{g} \mathrm{l}^{-1} \mathrm{CdCl}_{2}$ was added to the nutrient solution. Vertical bars represent the standard deviations of four plants. Letters above the bars indicate the level of significance at $p<0.05$ using the Tukey method.

evaluated the translocation ratios of copper $(\mathrm{Cu})$, iron $(\mathrm{Fe})$, magnesium $(\mathrm{Mg})$, manganese $(\mathrm{Mn})$, and $\mathrm{Zn}$. As shown in Figure 3, even in line R3 in which OsHMA3 was strongly suppressed, no obvious differences were observed compared to the control. This result suggests that OsHMA3 is a Cd-specific transporter, as reported in previous studies (Miyadate et al. 2011; Ueno et al. 2010).

After confirming the function of OsHMA3 and providing evidence that a construct formed by the fusion of the $5^{\prime}$ - and $3^{\prime}$-UTR of the OsHMA3 gene downregulates its expression, we next used the OsHMA3 RNAi plants acquired above to analyze the relationship between OsHMA2 and OsHMA3 in plant. Our original materials for the analysis were oshma2 mutants and 'CKK'. As the oshma2 mutants were in the 'Nippponbare' genetic background and 'CKK' is an indica cultivar, once we made crosses between oshma2 mutants and 'CKK' the $\mathrm{F}_{1}$ plants showed heterosis; vegetative growth continued for an extremely long time and the plants were nearly sterile. Although we harvested some $\mathrm{F}_{2}$ seeds, the $\mathrm{F}_{2}$ progeny showed phenotypic variation (e.g., variable plant height). Evaluating the function of OsHMA3 and OsHMA2 from such plants appeared to be difficult. In this experiment, OsHMA3 RNAi plants were suitable materials for combining with oshma2 mutants because all of the plants were in the 'Nipponbare' genetic 

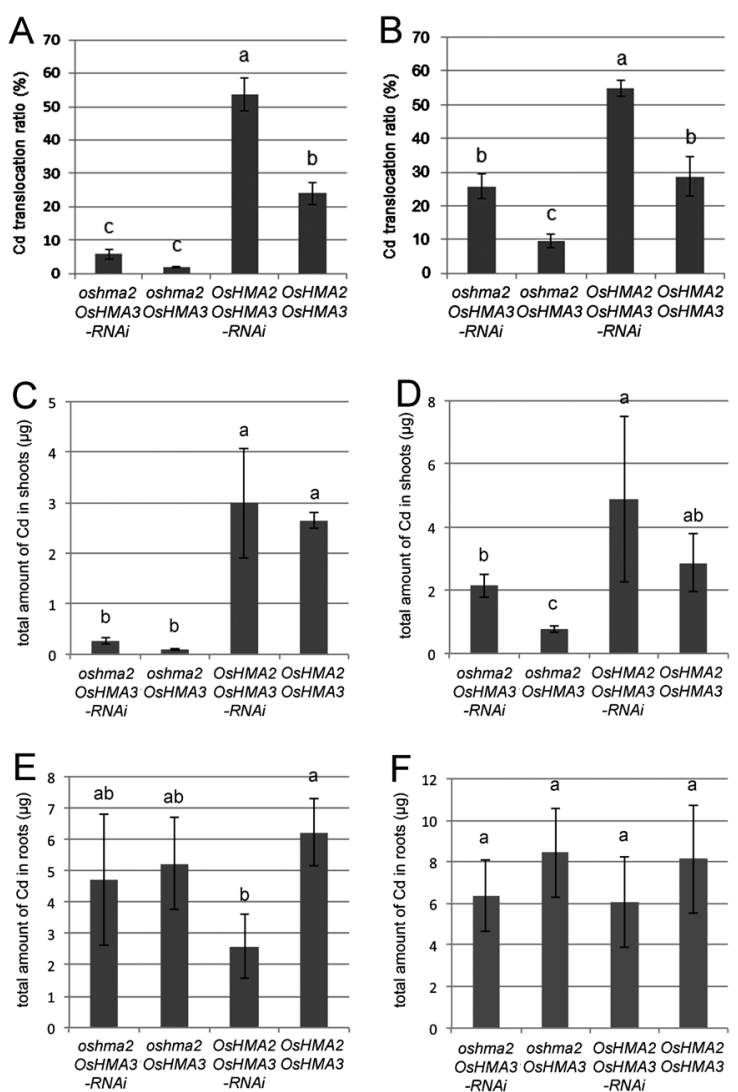

Figure 4. Cd translocation ratio and total amount of $\mathrm{Cd}$ in shoots and roots in oshma2-1 OsHMA3-RNAi, oshma2-1 OsHMA3, OsHMA2 OsHMA3-RNAi, and OsHMA2 OsHMA3. The oshma2-1 allele was used for the analysis, respectively, in (A), (C) and (E) and the oshma2-2 allele was used for the analysis, respectively, in (B), (D) and (F). Vertical bars represent the standard deviations of analyzed plants. Letters above the bars indicate the level of significance at $p<0.05$ using the Tukey method.

\section{background.}

Prior to making the crosses, the lines were genotyped to verify the presence of oshma2 mutations or OsHMA3 RNAi constructs. Genotyping for the Tos 17 insertions in oshma2 mutants was performed according to the method of Satoh-Nagasawa et al. (2012). Genotyping for OsHMA3 RNAi constructs was conducted as described above. For the oshma2 mutants, we used oshma2-1 and oshma2-2. In oshma2-1, more than half of the OsHMA2 protein was lost, with very little activity remaining; in oshma2-2, the C-terminal region of the OsHMA protein was missing, but some function still remained (Satoh-Nagasawa et al. 2012). The R3 line of OsHMA3 RNAi plants was used because all plants carrying the construct showed suppressed expression of OsHMA3 in the $T_{1}$ generation, and the suppression was strongest among the lines we created (Figure 2). Heterozygous oshma2 mutants were fertilized with pollen from the OsHMA3 RNAi line R3 plants. The Cd translocation ratios were analyzed in the oshma2 homozygote mutants with the OsHMA3 RNAi construct (oshma2 oshma3), the oshma2 homozygous mutants without the OsHMA3
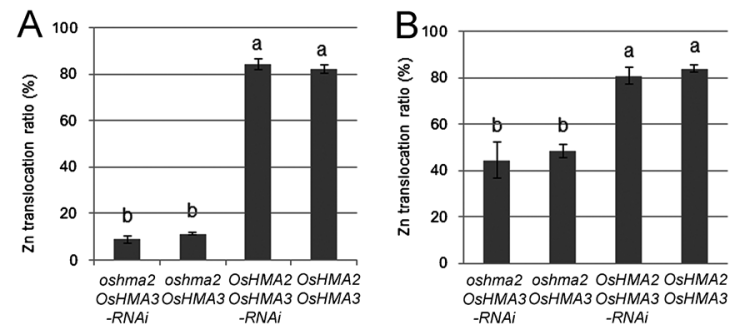

Figure 5. Zn translocation ratio in oshma2-1 OsHMA3-RNAi, oshma2-1 OsHMA3, OsHMA2 OsHMA3-RNAi, and OsHMA2 OsHMA3. The oshma2-1 allele was used for the analysis in (A) and the oshma2-2 allele was used for the analysis in (B). Vertical bars represent the standard deviations of analyzed plants. Letters above the bars indicate the level of significance at $p<0.05$ using the Tukey method.

RNAi construct (oshma2 OsHMA3), plants without the oshma2 mutations carrying the OsHMA3 RNAi construct (OsHMA2 OsHMA3-RNAi), and plants with neither the oshma2 mutation nor the OsHMA3 RNAi construct (OsHMA2 OsHMA3) in the $\mathrm{F}_{2}$ progeny.

Among 47 plants of the $\mathrm{F}_{2}$ population in which the presence of the OsHMA3 RNAi construct and the oshma2-1 mutation were confirmed, six plants of oshma2 OsHMA3-RNAi, four plants of OsHMA2 OsHMA3$R N A i$, six plants of oshma2 OsHMA3, and four plants of OsHMA2 OsHMA3 were selected and treated with $\mathrm{Cd}$. The $\mathrm{Cd}$ translocation ratio and total amount of $\mathrm{Cd}$ in shoots between oshma2-1 OsHMA3-RNAi and oshma2-1 OsHMA3 plants was not statistically different, whereas those of OsHMA2 OsHMA3-RNAi were higher than those of OsHMA2 OsHMA3 (Figures 4A, C). We speculate that loss of OsHMA3 function leads to accmulation of $\mathrm{Cd}$ outside of vacuole in roots temporary. If OsHMA2 activity was completely lost, Cd was not translocated from roots to shoots. On the other hand, if OsHMA2 functioned normally, Cd translocated. As for total amount of $\mathrm{Cd}$ in roots, there were no obvious difference between four genotypes (Figure 4E). Moreover, the result also implied that the activities of genes other than OsHMA2 are minor in loading Cd into xylem.

The same analysis of 49 plants of the $\mathrm{F}_{2}$ population in which the existence of the OsHMA3 RNAi construct and the oshma2-2 mutation were confirmed revealed that genotype oshma2-2 OsHMA3-RNAi had a similar Cd translocation ratio and total amount of $\mathrm{Cd}$ to OsHMA2 OsHMA3 rather than oshma2-2 OsHMA3 (Figures 4B, D). This means that the activity of mutated OsHMA2 is accelerated under high $\mathrm{Cd}$ concentrations in the roots even when the protein has lost its C-terminal region, which results in partial OsHMA2 activity. AtHMA2 ATPase activity is dependent on the metal concentration in vitro (Eren and Arguello 2004), whereas our findings demonstrated the accelerated activity of OsHMA2 in plant. In addition, we aquired information about the region that is important for regulating protein activity.

Because OsHMA2 also transports $\mathrm{Zn}$, we analyzed the 
$\mathrm{Zn}$ translocation ratio in the plants described above. The Zn translocation ratios of oshma2 OsHMA3-RNAi and oshma2 OsHMA3 were similar, and those of OsHMA2 OsHMA3-RNAi and OsHMA2 OsHMA3 were also similar (Figures 5A, B). That is, the genotype of OsHMA2 is crutial for $\mathrm{Zn}$ translocation. If OsHMA3 involve $\mathrm{Zn}$ transport into vacuoles, the genotype of OsHMA3 should also affect $\mathrm{Zn}$ translocation. Thus, here, we confirmed that OsHMA3 does not transport Zn.

As a whole, the phenotypes of oshma2 OsHMA3$R N A i$ plants in this study verified the sequential function of OsHMA3 and OsHMA2 in plant; OsHMA3 transports only Cd into vacuoles (Miyadate et al. 2011; Ueno et al. 2010) and OsHMA2 transports Cd and Zn into the xylem of rice roots (Nocito et al. 2011; SatohNagasawa et al. 2012; Takahashi et al. 2012). Moreover, our results suggest the importance of the complete loss of function of OsHMA2 to minimize Cd translocation from the roots to shoots. Recently, much attention has been paid to OsNRAMP5 and OsHMA3 for creating low and high heavy metal-accumulating rice for food and phytoextraction, respectively (Ishikawa et al. 2012; Miyadate et al. 2011; Ueno et al. 2010). Besides thse genes about $\mathrm{Cd}$ accmulation in rice breeding, we propose that much remains to be learned about the functional relationships of other genes in plant for the practical application of such resesarch, and that the combination of RNAi plants and mutants is a valuable way to analyze these relationships.

\section{Acknowledgements}

This work was supported by the Naito Foundation and by the Ministry of Education, Culture, Sports, Science and Technology, Japan (No. 21780007). We thank Dr. Takashi Sato for technical advice in operating inductively coupled plasma atomic emission spectrometry, Dr. Shohab Youssefian for technical advice about RTPCR, and Dr. Hiroki Rai for technical advice in the analysis of Cd concentration.

\section{References}

Clemens S, Aarts MG, Thomine S, Verbruggen N (2012) Plant Science: the key to preventing slow cadmium poisoning. Trends Plant Sci 18: 92-99

Eren E, Arguello JM (2004) Arabidopsis HMA2, a divalent heavy metal-transporting $\mathrm{P}_{1 \mathrm{~B}}$-type ATPase, is involved in cytoplasmic $\mathrm{Zn}^{2+}$ homeostasis. Plant Physiol 136: 3712-3723

Hiei Y, Ohta S, Komari T, Kumashiro T (1994) Efficient transformation of rice (Oryza sativa L.) mediated by Agrobacterium and sequence analysis of the boundaries of the T-DNA. Plant J 6: 271-228

Hussain D, Haydon MJ, Wang Y, Wong E, Sherson SM, Young J, Camakaris J, Harper JF, Cobbett CS (2004) P-type ATPase heavy metal transporters with roles in essential zinc homeostasis in Arabidopsis. Plant Cell 16: 1327-1339

Ishikawa S, Ishimaru Y, Igura M, Kuramata M, Abe T, Senoura T, Hase Y, Arao T, Nishizawa NK, Nakanishi H (2012) Ion-beam irradiation, gene identification, and marker-assisted breeding in the development of low-cadmium rice. Proc Natl Acad Sci USA 109: 19166-19171

Ishimaru Y, Takahashi R, Bashir K, Shimo H, Senoura T, Sugimoto K, Ono K, Yano M, Ishikawa S, Arao T, et al. (2012) Characterizing the role of rice NRAMP5 in manganese, iron and cadmium transport. Sci Rep 2: 286

Klimyuk VI, Carroll BJ, Thomas CM, Jones JDG (1993) Alkali treatment for rapid preparation of plant material for reliable PCR analysis. Plant J 3: 493-494

Mendoza-Cozatl D, Jobe T, Hauser F, Schroeder J (2011) Longdistance transport, vacuolar sequestration, tolerance, and transcriptional responses induced by cadmium and arsenic. Curr Opin Plant Biol 14: 554-562

Miki D, Itoh R, Shimamoto K (2005) RNA silencing of single and multiple members in a gene family of rice. Plant Physiol 138: 1903-1913

Miki D, Shimamoto K (2004) Simple RNAi vectors for stable and transient suppression of gene function in rice. Plant Cell Physiol 45: 490-495

Milner M, Kochian L (2008) Investigating heavy-metal hyperaccumulation using Thlaspi caerulescens as a model system. Ann Bot (Lond) 102: 3-13

Miyadate H, Adachi S, Hiraizumi A, Tezuka K, Nakazawa N, Kawamoto T, Katou K, Kodama I, Sakurai K, Takahashi H, et al. (2011) OsHMA3, a P1B-type of ATPase affects root-to-shoot cadmium translocation in rice by mediating efflux into vacuoles. New Phytol 189: 190-199

Mohanty A, Luo A, DeBlasio S, Ling X, Yang Y, Tuthill D, Williams K, Hill D, Zadrozny T, Chan A, et al. (2009) Advancing cell biology and functional genomics in maize using fluorescent protein-tagged lines. Plant Physiol 149: 601-605

Nocito FF, Lancilli C, Dendena B, Lucchini G, Sacchi GA (2011) Cadmium retention in rice roots is influenced by cadmium availability, chelation and translocation. Plant Cell Environ 34: 994-1008

Nriagu JO, Pacyna JM (1988) Quantitative assessment of worldwide contamination of air water and soils by trace metals. Nature 333: 134-139

Sasaki A, Yamaji N, Yokosho K, Ma JF (2012) Nramp5 is a major transporter responsible for manganese and cadmium uptake in rice. Plant Cell 24: 2155-2167

Satoh-Nagasawa N, Mori M, Nakazawa N, Kawamoto T, Nagato Y, Sakurai K, Takahashi H, Watanabe A, Akagi H (2012) Mutations in rice (Oryza sativa) heavy metal ATPase 2 (OsHMA2) restrict the translocation of zinc and cadmium. Plant Cell Physiol 53: 213-224

Takahashi R, Ishimaru Y, Shimo H, Ogo Y, Senoura T, Nishizawa NK, Nakanishi H (2012) The OsHMA2 transporter is involved in root-to-shoot translocation of $\mathrm{Zn}$ and $\mathrm{Cd}$ in rice. Plant Cell Environ 35: 1948-1957

Ueno D, Yamaji N, Kono I, Huang C, Ando T, Yano M, Ma JF (2010) Gene limiting cadmium accumulation in rice. Proc Natl Acad Sci USA 107: 16500-16505

Verbruggen N, Hermans C, Schat H (2009) Molecular mechanisms of metal hyperaccumulation in plants. New Phytol 181: 759-776

Wei S, Zhou Q (2008) Trace elements in agro-ecosystems. In: Prasad MVV (ed) Trace Elements as Contaminants and Nutrients-Consequences in Ecosystems and Human Health. Wiley, New Jersey, pp 55-80 